\title{
Demanda de atención obstétrica y densidad de recursos en salud para mujeres mexicanas en edad fértil
}

\author{
Javier Valdés-Hernández, ${ }^{1 *}$ Aldelmo E. Reyes-Pablo, ${ }^{2}$ Eduardo Navarrete-Hernández y \\ Sonia Canún-Serrano 4 \\ ${ }^{1}$ Secretaría de Salud, Hospital Nacional Homeopático, Servicio de Epidemiología; ${ }^{2}$ Secretaría de Salud, Hospital General "Dr. Manuel Gea \\ González", División de Gineco-obstetricia; ${ }^{3}$ Instituto Mexicano del Seguro Social, Coordinación de Vigilancia Epidemiológica, División de \\ Epidemiología Hospitalaria; ${ }^{4}$ Secretaría de Salud, Hospital General "Dr. Manuel Gea González", División de Genética Médica. Ciudad de México, \\ México
}

\section{Resumen}

Introducción: México registra aumento de las cesáreas e inequidad y desigualdad en la distribución de recursos para la atención obstétrica. Objetivo: Identificar las entidades y municipios en México que concentran la demanda de atención obstétrica y tasas de cesáreas y su relación con los recursos en salud y mujeres en edad fértil (MEF). Método: Se registraron los nacimientos del periodo 2008-2017, agrupados en cinco estratos municipales, y los recursos en salud y MEF de 2017. Resultados: La tasa nacional de cesáreas 2008-2017 fue de 45.3/100 nacimientos; 95 y 97 \% de los nacimientos y cesáreas se concentraron en el estrato "muy alto" (470 municipios), en el cual se utilizó 80 \% o más de los recursos en salud y destacó la sobreutilización. La densidad de recursos en salud destinados a las MEF reflejó inequidad y desigualdad. Conclusiones: La alta concentración de la demanda obstétrica y oferta de los recursos en salud pudiera conllevar mayor recurrencia a la cesárea. En las políticas de reducción de cesáreas es necesario considerar la organización y administración adecuadas de los recursos en salud.

PALABRAS CLAVE: Servicios de atención materna. Cesáreas. Recursos en salud. Mujer en edad fértil.

\section{Demand for obstetric care and density of health resources for childbearing age Mexican women}

\begin{abstract}
Introduction: In Mexico, there is an increase in the number of $C$-sections, as well as inequity and inequality in the distribution of resources for obstetric care. Objective: To identify the states and municipalities in Mexico that concentrate the demand for obstetric care and the $C$-section rates and their relationship with health resources and women of childbearing age (WCBA). Method: Births of the 2008-2017 period were recorded, grouped into five municipal strata, as well as 2017 health resources and WCBA. Results: The 2008-2017 national rate of C-sections was 45.3/100 births; 95 and $97 \%$ of births and C-sections were concentrated in the "very high" stratum, where $80 \%$ or more of health resources were used, with overuse standing out. The density of health resources assigned to WCBAs reflected inequity and inequality. Conclusions: The high concentration of obstetric demand and health resources supply could entail a higher recurrence of $C$-sections. Policies for $C$-section reduction should consider proper organization and administration of health resources.
\end{abstract}

KEY WORDS: Maternal health services. C-sections. Health resources. Woman of childbearing age. 


\section{Introducción}

Existen dos vías por las cuales se resuelve un nacimiento: vaginal o cesárea. Independientemente de la vía, hay un riesgo natural para la madre y el feto, por lo que el obstetra debe juzgar en cada caso qué opción es la mejor para ambos. ${ }^{1,2}$

La cesárea es la incisión en las paredes abdominal y uterina que tiene por objeto extraer el feto vivo o muerto de 22 o más semanas de gestación, así como la placenta y sus anexos; es la operación quirúrgica con más frecuencia en los servicios de obstetricia y su indicación es una responsabilidad en la práctica diaria del especialista en esa área..$^{3,4}$

Las indicaciones para cesárea son precisas y su único objetivo es asegurar la salud del binomio madre-hijo, por lo que este procedimiento quirúrgico solo debe realizarse en mujeres con parto de alto riesgo. ${ }^{2,5,6}$ Cuando se lleva a cabo sin que se cumplan las indicaciones precisas, los riesgos sobrepasan los beneficios, lo que genera complicaciones para las mujeres, violaciones a sus derechos reproductivos y costos adicionales para el sistema de salud.,8

La tasa de cesáreas (TC) es un indicador multidimensional del desempeño hospitalario, evalúa la atención médica adecuada, la seguridad madre-hijo y la eficiencia en la utilización de los recursos. Según la Organización Mundial de la Salud, la TC necesaria se estima entre 10 y $15 \%$ de los partos y la TC para disminuir los riesgos de salud materna-neonatal, entre 15 y $20 \%$.

Desde el 2000 se ha declarado el aumento de la TC en México y sus factores relacionados. ${ }^{9-12}$ En relación con los factores clínicos, en una revisión de 3232 expedientes del Instituto Mexicano del Seguro Social correspondientes al periodo 1997-1999, se señalaron 434 expresiones diagnósticas, heterogeneidad que contrastaba con la normatividad institucional vigente de 59 indicaciones específicas. ${ }^{13}$

Se han mencionado TC diferenciales al comparar las entidades federativas según su grado de marginación; las mayores tasas se encuentran en asociación positiva según el desarrollo socioeconómico: a menor marginación, TC superior y viceversa. ${ }^{14}$

Estudios acerca de la organización de los servicios de salud han encontrado que las TC son más altas en el sector privado que en el público y que en este último, la TC es mayor en las instituciones de seguridad social que en las dirigidas a población sin derechohabiencia. ${ }^{10,14}$
En México se ha señalado inequidad y desigualdad en la distribución de recursos en salud, en general y específicamente para la atención obstétrica. ${ }^{14-16}$

En cuanto al tamaño de los nosocomios, Campero et al. identificaron que la probabilidad de operación cesárea de una mujer demandante de atención obstétrica aumenta según el tamaño del hospital a partir de 10 camas. $^{17}$

Desde 1983, en México se han realizado varias reformas constitucionales para lograr el derecho a la protección de la salud y la cobertura universal de salud, sin embargo, las brechas de desigualdad persisten. Una de las causas es la oferta de recursos en salud, regida más por la demanda social de atención médica que por los requerimientos demográficos y de salud ${ }^{18}$ lo que deriva en divergencias en su utilización o eficiencia, estimada como el volumen de acciones por unidad de recursos en salud disponibles y la densidad de recursos para la salud (DRS), la cual se refiere a la razón entre los recursos que se disponen para satisfacer las necesidades de salud y la población blanco a la que están dirigidos, si bien no necesariamente estén disponibles en un momento dado para esa población. ${ }^{15}$

\section{Objetivos}

- Identificar las entidades federativas y municipios donde se concentra la mayor demanda de atención obstétrica, considerada a partir de los nacimientos registrados en el periodo 2008-2017 y las mayores TC, mediante la estratificación y el mapeo de la información.

- Analizar la relación estadística de los estratos municipales según la demanda de atención obstétrica y de cesáreas con la eficiencia de los recursos en salud en los municipios.

- Identificar los municipios donde se concentra el mayor número de mujeres en edad fértil de $15 \mathrm{a}$ 44 años (MEF) mediante la estratificación municipal y su relación estadística y geoespacial con la demanda de atención obstétrica y cesáreas y la DRS por municipio.

\section{Método}

Se calcularon las TC por entidades federativas y municipios conforme las bases de datos de nacimientos del Instituto Nacional de Estadística y Geografía (INEGI) y la Secretaría de Salud (SSa) para el periodo 2008-2017. Se tomaron en cuenta los 
nacimientos de 22 o más semanas de gestación y peso $\geq 500 \mathrm{~g}$.

Para el cálculo de la TC se tomó la entidad y municipio de nacimiento, lugar donde las mujeres gestantes demandaron y recibieron la atención del parto.

El análisis por entidad federativa de nacimiento y TC anuales no mostraron diferencias importantes, por lo que se acumuló la información del periodo; se procedió de la misma forma por municipios con el propósito de estabilizar las TC.

Para el resumen y análisis de los datos por entidades federativas y municipios, los nacimientos y las TC se agruparon en estratos: muy bajo $(\mathrm{MB})$, bajo $(B)$, medio (M), alto $(A)$ y muy alto (MA). El método de estratificación fue el de percentiles. La estratificación según el número de nacimientos se aprovechó como base de análisis para la agrupación de los datos restantes.

Tanto en las entidades federativas como en los municipios se realizó por separado un estudio de concordancia entre los estratos por nacimiento y cesárea, con la finalidad de identificar la relación entre las dos variables.

Para la mejor representación de algunos datos se empleó el mapeo. Para la elaboración de mapas se utilizó el programa Mapa Digital de México, versión 6.3.0, así como las capas cartográficas de las 32 entidades federativas del país y sus 2458 municipios elaboradas por el INEGI.

Los datos de los Recursos en Salud del año 2017 se adquirieron de la Dirección General de Información en Salud de la SSa y los de las MEF, de las proyecciones para ese año del Consejo Nacional de Población.

Con la finalidad de analizar la significación estadística en diferencias, se realizaron contrastes de medias de las TC por estratos mediante gráficas de error y análisis de varianza (Anova) para un factor. También se llevó a cabo chi cuadrada en la comparación de las TC por estratos.

\section{Resultados}

Los nacimientos con registros de entidades federativas de nacimiento fueron 20179539 (Figura 1). Los estratos MA y A concentraron $67.9 \%$ de los nacimientos, así como $69.5 \%$ de 9141552 cesáreas efectuadas en el periodo.

Se estimó una TC nacional de 45.3/100 nacimientos. Por entidades federativas, la TC osciló entre 31.2 y 52.2/100 nacimientos. Las cinco entidades federativas con mayores TC fueron Nuevo León, Yucatán, Sinaloa, Ciudad México y Tamaulipas; y las que obtuvieron menores TC fueron San Luis Potosí, Zacatecas, Nayarit, Chiapas y Durango.

Por estratos no se identificaron diferencias en las tasas, que fluctuaron de 42.5 a 47.6/100 nacimientos, no significativas estadísticamente según la gráfica de contraste de medias y el Anova. La gráfica de error mostró intervalos amplios de las TC por cada estrato, que se traslaparon con los contrarios. La chi cuadrada de las TC no mostró diferencias significativas entre estratos.

El contraste geoespacial de la información de nacimientos y de las TC indicó que las estratificaciones apenas alcanzaron una concordancia de $6.3 \%$ (Figura 1).

Los nacimientos con registro municipal fueron 20176197 (Figura 2). Hubo 109 (4.43\%) municipios sin datos.

De acuerdo con la estratificación, los nacimientos y las cesáreas se concentraron en el estrato MA, constituido por 470 municipios: 95 y $97 \%$, respectivamente. Además, la TC en ese estrato fue la más alta: 46.3/100 nacimientos. Las diferencias en las medias de TC por estratos son significativas estadísticamente, en particular al comparar el estrato MA con los restantes, según la gráfica de error y los resultados del Anova. Los estrechos intervalos de confianza en la gráfica de error por cada estrato indican cifras estables a su interior. La razón de tasas del estrato MA contra el A es de 1.58 y de 5.1 en comparación con el MB.

La concordancia entre municipios de nacimiento y TC fue de $55.3 \%$, sin embargo, el análisis por cada estrato dio como resultado que la concordancia entre los estratos MA de ambas agrupaciones fuera de $93 \%$. Existió una correlación espacial casi perfecta entre los municipios ubicados en el estrato MA de nacimientos con los del estrato MA en TC, lo que aumentó la confiabilidad de la tendencia a concentrar la demanda de atención obstétrica y las cesáreas en estos municipios.

En la Tabla 1 se describen los recursos en salud por estratos municipales según la demanda de atención obstétrica. En la mayoría se encontró una tendencia ascendente paralela a la estratificación de los nacimientos: de menos a más nacimientos, menos a más recursos en salud. Salvo 11 rubros, el estrato MA concentró $80 \%$ o más de los recursos. En los que tuvieron porcentajes menores a este límite, la 

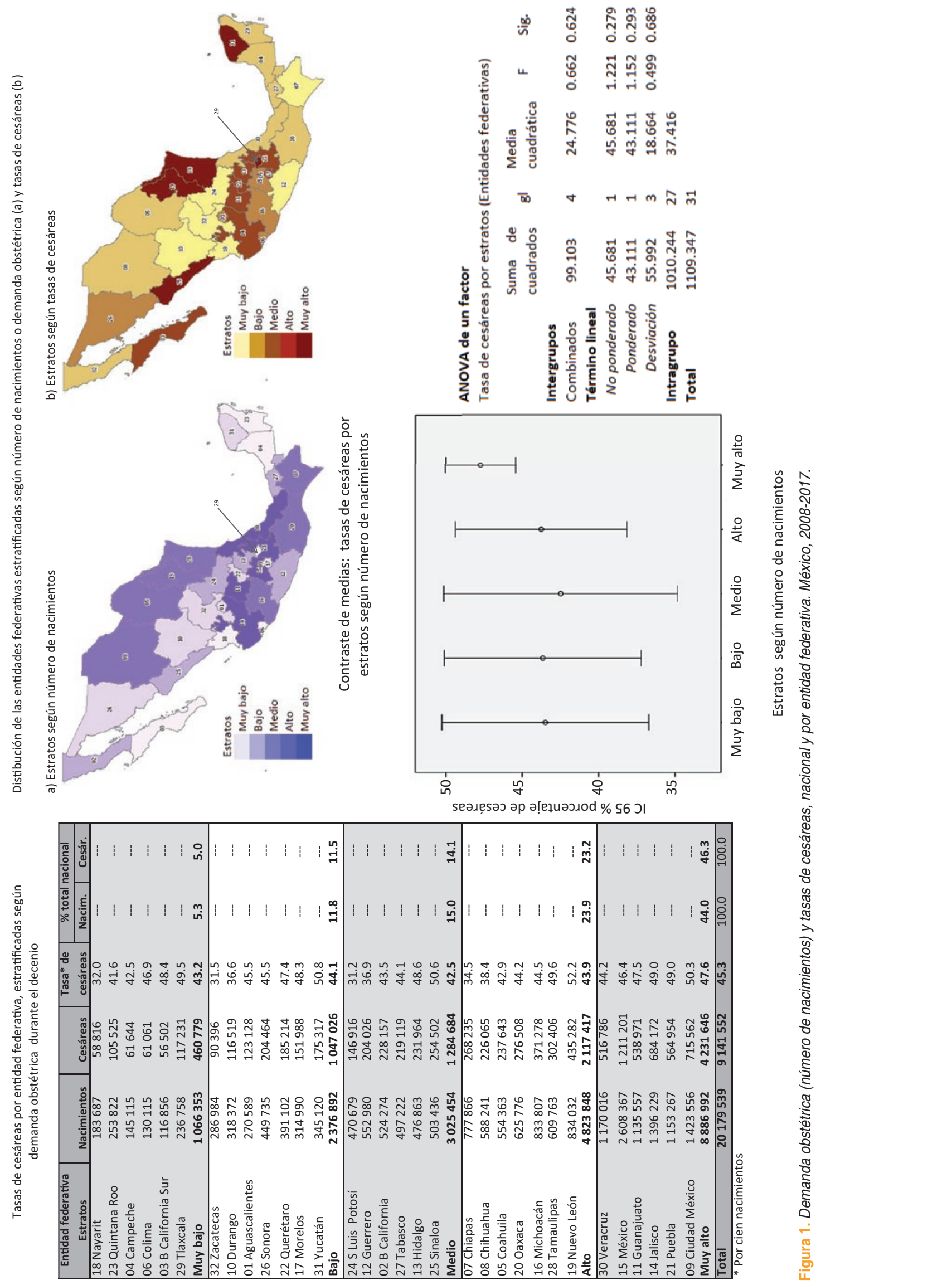


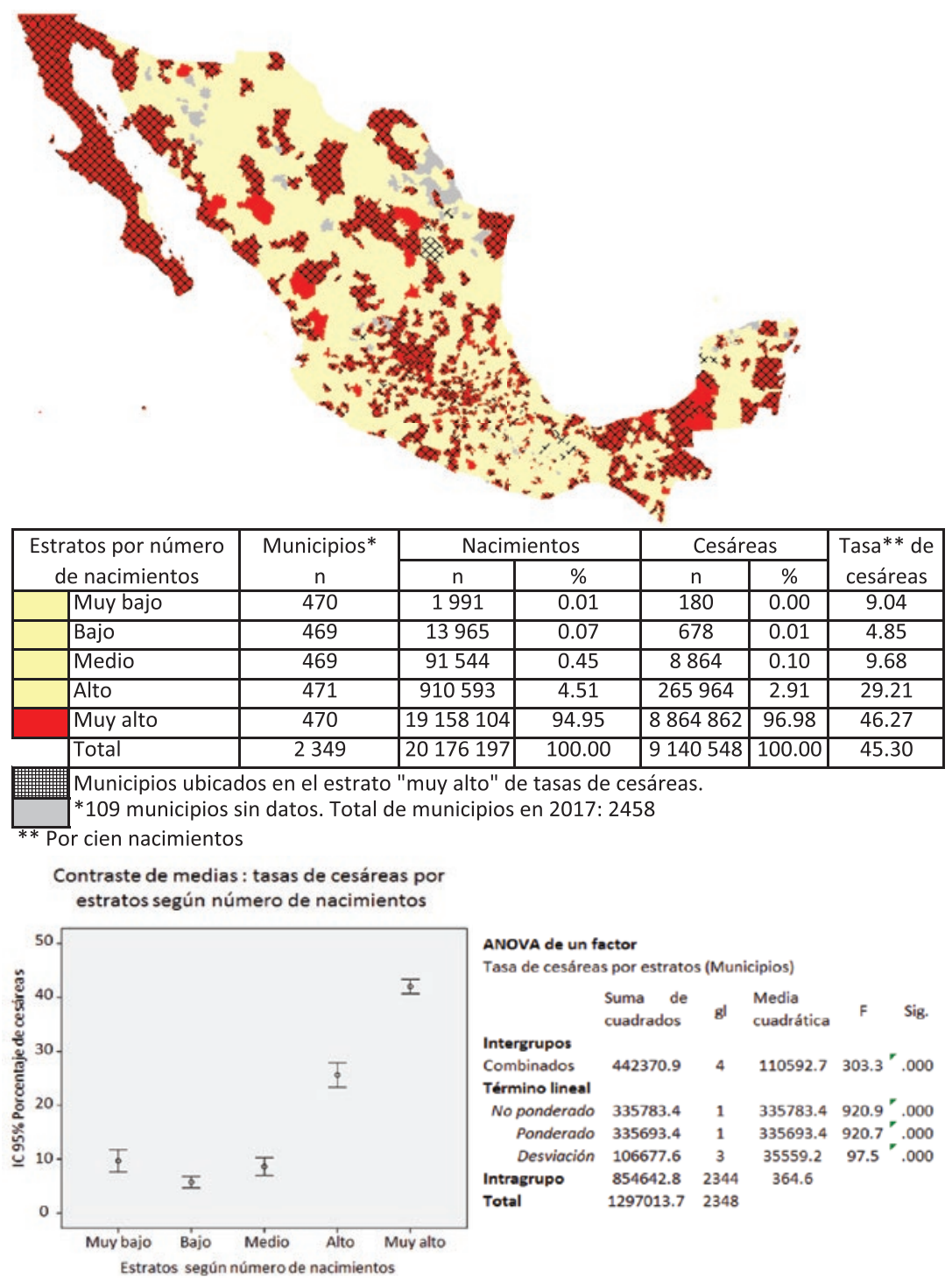

Figura 2. Distribución de la demanda obstétrica y tasas de cesáreas según estratos municipales y su coincidencia geoespacial. México, 20082017. Fuente: Bases de datos abiertas de nacimientos, INEGI/SSa, 2008-2017.

concentración fue igual o superior a $70 \%$ al sumar el estrato inmediatamente inferior, con predominio del estrato MA, lo cual confirma la tendencia de concentración de los recursos.

La Tabla 2 muestra la eficiencia de los recursos en salud. Excepto tres rubros en los nacimientos y dos en las cesáreas, se encontró un número ascendente de nacimientos o cesáreas por unidad paralela a la estratificación de los nacimientos: de menor a mayor estratificación de nacimientos, menos a más nacimientos o cesáreas por unidad de recursos en salud, lo que indica sobreutilización en el estrato MA y subutilización en el MB.

En cuanto a la distribución de las MEF por estratos según número de nacimientos (Figura 3), cerca de $75 \%$ de las MEF se ubicó en el estrato MA al que siguió el estrato $A$, que en conjunto sumaron aproximadamente $88 \%$. Esta distribución se ilustra geoespacialmente en el mapa: supera la extensión territorial de los 470 municipios donde se concentró $95 \%$ de los nacimientos.

Las mujeres que migraron para su atención en el estrato MA constituyeron $41.1 \%, 34.5 \%$ en el A y $29.6 \%$ en el MB. Las cifras de DRS para MEF tiene un comportamiento aleatorio, lo que refleja inequidad y desigualdad en el otorgamiento de los recursos; cifras similares indicarían equidad e igualdad.

\section{Discusión}

En la actualidad, los estudios ecológicos con niveles en áreas pequeñas son más convenientes por ser más 
Tabla 1. Distribución de los recursos en salud por estratos municipales según el número de nacimientos o demanda obstétrica. México, 2008-2017

\begin{tabular}{|c|c|c|c|c|c|c|c|c|c|c|c|}
\hline \multirow[t]{3}{*}{ Recursos en salud } & \multicolumn{11}{|c|}{ Estratos según número de nacimientos } \\
\hline & \multicolumn{2}{|c|}{ Muy bajo } & \multicolumn{2}{|c|}{ Bajo } & \multicolumn{2}{|c|}{ Medio } & \multicolumn{2}{|c|}{ Alto } & \multicolumn{2}{|c|}{ Muy alto } & \multirow[t]{2}{*}{ Total } \\
\hline & $\mathrm{n}$ & $\%$ & $\mathrm{n}$ & $\%$ & $\mathrm{n}$ & $\%$ & $\mathrm{n}$ & $\%$ & n & $\%$ & \\
\hline Unidades de primer nivel & 1228 & 5.9 & 1887 & 9.1 & 2969 & 14.4 & 4702 & 22.7 & 9883 & 47.8 & 20669 \\
\hline Unidades de segundo nivel & 2 & 0.2 & 6 & 0.5 & 20 & 1.5 & 232 & 17.7 & 1050 & 80.2 & 1310 \\
\hline Área de hospitalización & 2 & 0.1 & 9 & 0.5 & 29 & 1.7 & 260 & 14.8 & 1455 & 82.9 & 1755 \\
\hline Unidad quirúrgica & 0 & 0.0 & 1 & 0.1 & 12 & 1.0 & 208 & 17.7 & 951 & 81.1 & 1172 \\
\hline UCIN & 0 & 0.0 & 0 & 0.0 & 1 & 0.3 & 8 & 2.3 & 333 & 97.4 & 342 \\
\hline Área de tocología y tococirugía & 56 & 3.6 & 67 & 4.3 & 86 & 5.5 & 327 & 21.0 & 1021 & 65.6 & 1557 \\
\hline Área de neonatología y cuneros & 1 & 0.1 & 5 & 0.6 & 13 & 1.6 & 105 & 12.7 & 701 & 85.0 & 825 \\
\hline Quirófanos & 2 & 0.0 & 3 & 0.1 & 19 & 0.5 & 314 & 7.7 & 3714 & 91.7 & 4052 \\
\hline Salas de expulsión & 100 & 3.8 & 169 & 6.4 & 214 & 8.1 & 528 & 20.0 & 1632 & 61.7 & 2643 \\
\hline Cunas para recién nacido sano & 31 & 0.4 & 49 & 0.7 & 71 & 1.0 & 480 & 6.6 & 6599 & 91.3 & 7230 \\
\hline Total de consultorios & 1864 & 2.5 & 2832 & 3.8 & 4410 & 5.9 & 9016 & 12.2 & 56036 & 75.6 & 74158 \\
\hline Medicina general & 1508 & 3.9 & 2274 & 5.9 & 3457 & 8.9 & 6184 & 15.9 & 25355 & 65.4 & 38778 \\
\hline Ginecoobstetricia & 2 & 0.1 & 6 & 0.3 & 27 & 1.3 & 229 & 11.4 & 1746 & 86.9 & 2010 \\
\hline Pediatría & 3 & 0.2 & 4 & 0.2 & 26 & 1.5 & 186 & 10.7 & 1527 & 87.5 & 1746 \\
\hline Total camas área hospitalización & 15 & 0.0 & 250 & 0.3 & 570 & 0.7 & 4074 & 4.7 & 82014 & 94.4 & 86923 \\
\hline Medicina general & 3 & 0.2 & 6 & 0.4 & 42 & 2.9 & 540 & 36.9 & 874 & 59.7 & 1465 \\
\hline Ginecoobstetricia & 1 & 0.0 & 15 & 0.1 & 48 & 0.3 & 983 & 5.9 & 15533 & 93.7 & 16580 \\
\hline Cirugía general y reconstructiva & 0 & 0.0 & 6 & 0.0 & 32 & 0.2 & 643 & 4.6 & 13347 & 95.1 & 14028 \\
\hline Pediatría & 4 & 0.0 & 14 & 0.1 & 105 & 1.0 & 683 & 6.2 & 10131 & 92.6 & 10937 \\
\hline Área de labor de parto & 199 & 4.0 & 327 & 6.5 & 442 & 8.8 & 631 & 12.5 & 3438 & 68.3 & 5037 \\
\hline Área de recuperación posparto & 0 & 0.0 & 8 & 7.8 & 14 & 13.6 & 4 & 3.9 & 77 & 74.8 & 103 \\
\hline Total de médicos & 1078 & 2.1 & 1984 & 3.8 & 3528 & 6.7 & 8178 & 15.6 & 37605 & 71.8 & 52373 \\
\hline Generales & 189 & 1.0 & 210 & 1.1 & 261 & 1.4 & 824 & 4.3 & 17716 & 92.3 & 19200 \\
\hline Pediatras & 19 & 0.2 & 6 & 0.1 & 26 & 0.2 & 398 & 3.7 & 10356 & 95.8 & 10805 \\
\hline Ginecoobstetras & 14 & 0.2 & 3 & 0.0 & 31 & 0.3 & 408 & 4.5 & 8525 & 94.9 & 8981 \\
\hline Cirujanos & 14 & 0.2 & 3 & 0.0 & 7 & 0.1 & 128 & 1.8 & 6986 & 97.9 & 7138 \\
\hline Anestesiólogos & 2 & 0.1 & 18 & 1.1 & 10 & 0.6 & 12 & 0.8 & 1531 & 97.3 & 1573 \\
\hline En formación & 759 & 5.9 & 978 & 7.6 & 1235 & 9.6 & 2047 & 15.9 & 7854 & 61.0 & 12873 \\
\hline Residentes & 96 & 0.6 & 127 & 0.8 & 264 & 1.7 & 794 & 5.1 & 14339 & 91.8 & 15620 \\
\hline Pasantes & 96 & 2.7 & 185 & 5.2 & 269 & 7.5 & 353 & 9.9 & 2673 & 74.7 & 3576 \\
\hline Interno de pregrado & 0 & 0.0 & 1 & 0.0 & 9 & 0.0 & 232 & 0.9 & 24287 & 99.0 & 24529 \\
\hline Total de enfermeras & 740 & 0.5 & 1228 & 0.8 & 2682 & 1.8 & 7664 & 5.2 & 133671 & 91.6 & 145985 \\
\hline Generales & 75 & 0.2 & 86 & 0.2 & 224 & 0.6 & 781 & 2.2 & 34647 & 96.7 & 35813 \\
\hline Parteras & 1012 & 0.5 & 1354 & 0.7 & 2936 & 1.4 & 11220 & 5.4 & 191723 & 92.1 & 208245 \\
\hline
\end{tabular}


Tabla 2. Utilización de recursos en salud por estratos municipales según demanda obstétrica o número de nacimientos. México, 2008-2017

\begin{tabular}{|c|c|c|c|c|c|c|}
\hline \multirow[t]{3}{*}{ Recursos en salud } & \multicolumn{6}{|c|}{ Estratos según número de nacimientos } \\
\hline & Muy bajo & Bajo & Medio & Alto & Muy alto & Total \\
\hline & $\begin{array}{l}\text { Relación } \\
\text { RS/N o C }\end{array}$ & $\begin{array}{l}\text { Relación } \\
\text { RS/N o C }\end{array}$ & $\begin{array}{l}\text { Relación } \\
\text { RS/N o C }\end{array}$ & $\begin{array}{l}\text { Relación } \\
\text { RS/N o C }\end{array}$ & $\begin{array}{l}\text { Relación } \\
\text { RS/N o C }\end{array}$ & $\begin{array}{l}\text { Relación } \\
\text { RS/N o C }\end{array}$ \\
\hline Número de nacimientos & 1991 & 13965 & 91544 & 910593 & 19158104 & 20176197 \\
\hline Área de tocología y tococirugía & 36 & 208 & 1064 & 2785 & 18764 & 12958 \\
\hline Salas de expulsión & 20 & 83 & 428 & 1725 & 11739 & 7634 \\
\hline Consultorios de medicina general & 1 & 6 & 26 & 147 & 756 & 520 \\
\hline Consultorios de ginecoobstetricia & 996 & 2328 & 3391 & 3976 & 10973 & 10038 \\
\hline Camas de ginecoobstetricia & 1991 & 931 & 1907 & 926 & 1233 & 1217 \\
\hline Camas en área de labor de parto & 10 & 43 & 207 & 1443 & 5572 & 4006 \\
\hline Camas en recuperación posparto & 0 & 1746 & 6539 & 227648 & 248807 & 195885 \\
\hline Médicos generales & 11 & 67 & 351 & 1105 & 1081 & 1051 \\
\hline Ginecobstetras & 142 & 4655 & 2953 & 2232 & 2247 & 2247 \\
\hline Médicos anestesiólogos & 996 & 776 & 9154 & 75883 & 12513 & 12827 \\
\hline Pasantes de medicina & 21 & 75 & 340 & 2580 & 7167 & 5642 \\
\hline Internos de pregrado & 0 & 13965 & 10172 & 3925 & 789 & 823 \\
\hline Enfermeras & 3 & 11 & 34 & 119 & 143 & 138 \\
\hline Enfermería general & 27 & 162 & 409 & 1166 & 553 & 563 \\
\hline Parteras & 2 & 10 & 31 & 81 & 100 & 97 \\
\hline Número de cesáreas & 180 & 678 & 8864 & 265964 & 8864862 & 9140548 \\
\hline Área de tocología y tococirugía & 3 & 10 & 103 & 813 & 8683 & 5871 \\
\hline Salas de expulsión & 2 & 4 & 41 & 504 & 5432 & 3458 \\
\hline Consultorios de medicina general & 0 & 0 & 3 & 43 & 350 & 236 \\
\hline Consultorios de ginecoobstetricia & 90 & 113 & 328 & 1161 & 5077 & 4548 \\
\hline Camas de ginecoobstetricia & 180 & 45 & 185 & 271 & 571 & 551 \\
\hline Camas en área de labor de parto & 1 & 2 & 20 & 421 & 2578 & 1815 \\
\hline Camas en recuperación posparto & 0 & 85 & 633 & 66491 & 115128 & 88743 \\
\hline Médicos generales & 1 & 3 & 34 & 323 & 500 & 476 \\
\hline Ginecobstetras & 13 & 226 & 286 & 652 & 1040 & 1018 \\
\hline Médicos anestesiólogos & 90 & 38 & 886 & 22164 & 5790 & 5811 \\
\hline Pasantes de medicina & 2 & 4 & 33 & 753 & 3316 & 2556 \\
\hline Internos de pregrado & 0 & 678 & 985 & 1146 & 365 & 373 \\
\hline Enfermeras & 0 & 1 & 3 & 35 & 66 & 63 \\
\hline Enfermería general & 2 & 8 & 40 & 341 & 256 & 255 \\
\hline Parteras & 0 & 1 & 3 & 24 & 46 & 44 \\
\hline
\end{tabular}

Fuente: Bases de datos abiertas de nacimientos INEGI/SSA 2008-2017 y Recursos en Salud 2017 de la SSa. RS $=$ recursos en salud. $\mathrm{N}=$ nacimientos, $\mathrm{C}=$ cesáreas. 


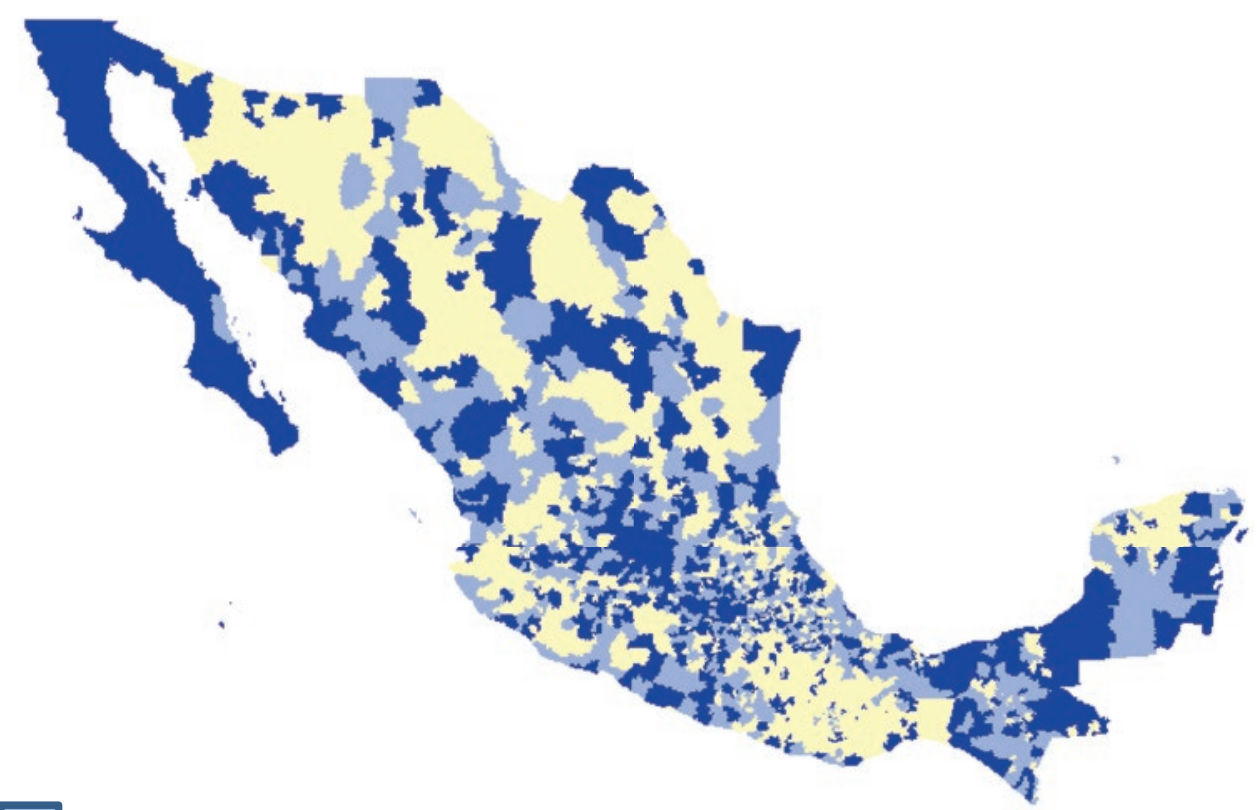

Municipios donde reside $88 \%$ de las mujeres de 15 a 44 años

Municipios donde se atendió $95 \%$ de los nacimientos en el periodo

\begin{tabular}{|c|c|c|c|c|c|c|}
\hline \multirow[t]{2}{*}{ Indicador } & \multicolumn{6}{|c|}{ Estratos según número de nacimientos } \\
\hline & Muy bajo & Bajo & Medio & Alto & Muy alto & Total \\
\hline $\begin{array}{l}\text { Mujeres de } 15 \text { a } 44 \text { años } \\
\quad \text { Porcentaje }\end{array}$ & $\begin{array}{c}620,148 \\
2.1\end{array}$ & $\begin{array}{c}1,122,688 \\
3.8\end{array}$ & $\begin{array}{c}1,883,577 \\
6.3\end{array}$ & $\begin{array}{l}3,900,111 \\
13.1\end{array}$ & $\begin{array}{c}22,350,537 \\
74.8\end{array}$ & $\begin{array}{c}29,877,062 \\
100.0\end{array}$ \\
\hline $\begin{array}{l}\text { Porcentaje de nacimientos con } \\
\text { residencia habitual diferente al del } \\
\text { municipo de atención }\end{array}$ & 29.6 & 20.6 & 18.3 & 34.5 & 41.1 & 40.7 \\
\hline Recursos en salud 2017 & \multicolumn{6}{|c|}{ Densidad de recursos en salud por mujeres de $15-44$ años de edad } \\
\hline Área de tocología y tococirugía & 11,074 & 16,757 & 21,902 & 11,927 & 21,891 & 19,189 \\
\hline Salas de expulsión & 6,201 & 6,643 & 8,802 & 7,387 & 13,695 & 11,304 \\
\hline Consultorios de medicina general & 411 & 494 & 545 & 631 & 882 & 770 \\
\hline Consultorios de ginecoobstetricia & 310,074 & 187,115 & 69,762 & 17,031 & 12,801 & 14,864 \\
\hline Camas de ginecoobstetricia & 620,148 & 74,846 & 39,241 & 3,968 & 1,439 & 1,802 \\
\hline Camas en área de labor de parto & 3,116 & 3,433 & 4,261 & 6,181 & 6,501 & 5,932 \\
\hline Camas en recuperación posparto & 0 & 140,336 & 134,541 & 975,028 & 290,267 & 290,069 \\
\hline Médicos generales & 3,281 & 5,346 & 7,217 & 4,733 & 1,262 & 1,556 \\
\hline Ginecoobstetras & 44,296 & 374,229 & 60,761 & 9,559 & 2,622 & 3,327 \\
\hline Médicos anestesiólogos & 310,074 & 62,372 & 188,358 & 325,009 & 14,599 & 18,994 \\
\hline Pasantes de medicina & 6,460 & 6,069 & 7,002 & 11,048 & 8,362 & 8,355 \\
\hline Internos de pregrado & 0 & $1,122,688$ & 209,286 & 16,811 & 920 & 1,218 \\
\hline Enfermeras & 838 & 914 & 702 & 509 & 167 & 205 \\
\hline Enfermería general & 8,269 & 13,055 & 8,409 & 4,994 & 645 & 834 \\
\hline \begin{tabular}{|l|} 
Parteras \\
\end{tabular} & 613 & 829 & 642 & 348 & 117 & 143 \\
\hline
\end{tabular}

Figura 3. Relación entre recursos en salud y mujeres de 15 a 44 años por estratos municipales según demanda obstétrica o número de nacimientos. México, 2008-2017. Fuente: Bases de datos abiertas de nacimientos, INEGI/SSa, 2008-2017, Recursos en Salud 2017 de la SSa y estimaciones de población 2017 del Consejo Nacional de Población.

esclarecedores. ${ }^{19}$ Con este enfoque, en nuestro análisis identificamos alta concentración de la demanda de atención obstétrica y de cesáreas en 470 municipios asociados con la conglomeración de los recursos en salud. No obstante, se observaron desigualdades en la utilización de los recursos en salud en los estratos $\mathrm{A}$ y MA, con sobreuso por la excesiva demanda de atención obstétrica. Respecto a los recursos humanos en salud, esta relación puede considerarse como un indicador de productividad. En cuanto a los resultados, la sobresaturación de los servicios de ginecoobstetricia pudiera conllevar a mayor recurrencia a la operación cesárea como una estrategia para liberar camas en las salas de labor, considerando el tiempo efectivo de cuidados requerido por un parto en comparación con la cesárea. 
Desde su conformación en la década de 1950, el modelo médico mexicano está centrado en la atención curativa o resolutiva ${ }^{20}$ que ubica las salas de atención del parto en los hospitales, privilegiando al segundo y tercer nivel sobre el primero, transformando así la práctica obstétrica en un asunto de "especialidad médica" en forma paralela al proceso de desplazamiento de la medicina general: existen 1.7 médicos especialistas por cada médico general (Tabla 1).

El $98 \%$ de los nacimientos en el periodo de estudio fue atendido en hospitales; $63 \%$ de las áreas de tocología y tococirugía, $93 \%$ de las áreas de neonatología y cuneros, $95 \%$ de los quirófanos, $56 \%$ de las salas de expulsión y $93 \%$ de las cunas para recién nacidos sanos se ubican en el segundo nivel. Por el contrario, $92 \%$ de los consultorios de medicina general, $83 \%$ de los médicos generales y $3 \%$ de los especialistas en medicina familiar se ubican en unidades de primer nivel.

Los resultados confirman la política sanitaria distributiva implementada en México, la cual soslaya el criterio de la población usuaria en potencia para la dotación de los servicios. En este estudio se destacó la DRS desigual en relación con el número de MEF, lo cual podría explicar el alto porcentaje de migración intermunicipal de las gestantes para la atención del nacimiento hacia los municipios con mayores recursos o disponibles en el momento en que los requerían. Dos de cada cinco nacimientos ocurrieron en localidad distinta a la residencia habitual de la madre. Aunque la densidad local de recursos en los estratos de nacimiento $B$ y MB fue mejor, existió un porcentaje alto de atención en otros municipios, ya fuera porque en estos se concentraban los recursos en salud o porque los servicios de atención obstétrica locales no laboraban al momento de la demanda.

De acuerdo con los resultados, a los factores conocidos asociados con altas TC se agrega la planeación inadecuada de los recursos en salud para la atención obstétrica y el modelo de atención médica, por lo que se requiere considerar también estas dimensiones en el desarrollo de políticas públicas encaminadas a la reducción de las tasas de TC.

En esta reingeniería sería necesario diseñar las unidades de primer nivel con un área aceptable para la atención de partos inminentes o de bajo riesgo, considerando la distribución de la población demandante y los recursos en salud necesarios, además de fortalecer el primer nivel de atención, lo cual requiere también mejorar las capacidades de los médicos generales y la calidad de la atención.

Adicionalmente, es necesario estimar una TC adecuada y racional al contexto del país, que sirva de referencia para las evaluaciones ulteriores sobre cesáreas, bajo la normatividad vigente de la atención del parto. Además, se requieren indicadores racionales de productividad y utilización de los recursos de salud.

La modificación del perfil demográfico y epidemiológico de la población de México son previsibles para los próximos años. Se necesita que el sistema de salud se ajuste para dar respuesta a estos cambios, incluidos fundamentalmente la composición de los recursos humanos, en particular hacia la atención obstétrica por vía vaginal.

El enfoque del presente reporte de investigación ofrece conclusiones novedosas e importantes en el contexto que vive el país.

\section{Agradecimientos}

A los ginecoobstetras Josué Jáuregui Valdez y Didilia Elena Bejarano Velázquez, por sus comentarios sobre las cesáreas en hospitales públicos, que enriquecieron este trabajo.

\section{Bibliografía}

1. American College of Obstetricians and Gynecologists; Society for Maternal-Fetal Medicine; Caughey AB, Cahill AG, Guise JM, Rouse DJ. Safe prevention of the primary cesarean delivery. Am J Obstet Gynecol. 2014;210:179-193.

2. Villanueva-Egan LA. Operación cesárea: una perspectiva integral. Rev Fac Med UNAM. 2004;47:246-250.

3. Lee SI. Operación cesárea: estudio de causas y tendencias en un hospital de segundo nivel. Rev Med IMSS. 2004;42:199-204.

4. Muñoz-Enciso JM, Rosales-Aujang E, Domínguez-Ponce G, Serrano-Díaz CL. Operación cesárea: ¿indicación justificante o preocupación justificada? Ginecol Obstet Mex. 2011;79:67-74.

5. Guía de práctica clínica. Reducción de la frecuencia de operación cesárea México: Instituto Mexicano del Seguro Social; 2014.

6. Suárez-López L, Campero L, de la Vara-Salazar E, Rivera-Rivera L, Hernández-Serrato MI, Walker D, et al. Características sociodemográficas y reproductivas asociadas con el aumento de cesáreas en México. Salud Publica Mex. 2013;55:S225-S234.

7. Declaración sobre tasas de cesárea. Suiza: Organización Mundial de la Salud/Departamento de Salud Reproductiva e Investigación; 2015

8. Rosales-Aujang E, Felguérez-Flores J. Repercusión demográfica de la operación cesárea. Ginecol Obstet Mex. 2009;77:362-366

9. González-Pérez GJ, Vega-López MG, Cabrera-Piraval C, Muñoz A, VaIle A. Caesarean sections in Mexico: are there too many? Health Policy Plan. 2001;16:62-67.

10. Suárez L, Campero L, de la Vara E, Rivera L, Hernández MI, Walker D, et al. Elevada recurrencia a las cesáreas: revertir la tendencia y mejorar la calidad en el parto. México: Instituto Nacional de Salud Pública; 2019

11. Gómez-Dantés $O$, Sesma S, Becerril VM, Knaul FM, Arreola H, Frenk J. Sistema de salud de México. Salud Publica Mex. 2011;53: S220-S232.

12. Beltrán AP, Ye J, Moller AB, Zhang J, Gülmezoglu AM, Torloni MR. The increasing trend in caesarean section rates: global, regional and national estimates: 1990-2014. PLoS One. 2016;11:e0148343. 
13. Velasco-Murillo V, Navarrete-Hernández E, Pozos-Cavanzo JL, Ojeda-Mijares RI, Cárdenas-Lara C, Cardona-Pérez JA. Indicaciones y justificación de las cesáreas en el Instituto Mexicano del Seguro Social. Gac Med Mex. 2000;136:421-431.

14. González-Pérez GJ, Vega-López MG, Cabrera-Piraval CE. Cesáreas en México: aspectos sociales, económicos y epidemiológicos. México: Centro Universitario de Ciencias de la Salud; 2011.

15. Ochoa-Moreno JA. Densidad de recursos para la atención de la salud de la población no derechohabiente en México, en 2013. México: Organización Panamericana de la Salud/Organización Mundial de la Salud/ Secretaría de la Salud/Comisión Nacional de Arbitraje Médico; 2016.

16. Alcalde-Rabanal JE, Nigenda G, Serván-Mori E, González-Robledo LM Lozano R. Brechas en la disponibilidad de médicos y enfermeras especialistas en el sistema nacional de salud. Informe Final. México: Instituto Nacional de Salud Pública/Comisión Nacional de Seguridad/ Dirección General de Calidad y Educación en Salud/Comité de Estudio de Necesidades de Formación de Recursos Humanos en Salud/Comisión Interinstitucional para la Formación de Recursos Humanos para la Salud; 2017.

17. Campero L, Hernández B, Leyva A, Estrada F, Osborne J, Morales S. Tendencias de cesáreas en relación con factores no clínicos en un centro de educación para el parto en la Ciudad de México. Salud Publica Mex. 2007; 49:118-125.

18. Hernández-Palacios RD. Una prospectiva de la salud en México. Algunos aspectos del marco sociojurídico. Alegatos. 2007;65:47-56.

19. Silva-Ayçaguer LC, Benavides-Rodríguez A, Vidal-Rodeiro CL. Análisis espacial de la mortalidad en áreas geográficas pequeñas. El enfoque bayesiano. Rev Cubana Salud Publica. 2003;29:314-322.

20. Hernández-Llamas H. Historia de la participación del Estado en las instituciones de atención médica en México, 1935-1980. En: Ortiz-Quesada F, editor. Vida y muerte del mexicano. México: Ediciones Folios; 1982 